\title{
First comparison of primary frequency standards between Europe and Asia
}

\author{
M. Fujieda*, T. Gotoh*, D. Piester ${ }^{\dagger}$, M. Kumagai*, S. Weyers ${ }^{\dagger}$, A. Bauch ${ }^{\dagger}$, R. Wynands ${ }^{\dagger}$ and M. Hosokawa* \\ *National Institute of Information and Communications Technology, Koganei 184-8795, Japan (e-mail: miho@nict.go.jp) \\ ${ }^{\dagger}$ Physikalisch-Technische Bundesanstalt, Braunschweig, Germany
}

\begin{abstract}
In December 2006, PTB and NICT simultaneously operated their caesium atomic fountain primary frequency standards, PTB-CSF1 and NICT-CsF1 for 15 days starting from MJD 54079. This was the first direct comparison of primary frequency standards between Europe and Asia. The fountains were compared to local H-masers and the frequency difference between the H-masers was determined directly by TWSTFT with frequency transfer uncertainty below $10^{-15}$. PTB-CSF1 and NICT-CsF1 showed good agreement within the stated uncertainties.
\end{abstract}

\section{INTRODUCTION}

Recently, research and development of primary frequency standards has advanced and their uncertainties have reached the level of one part in $10^{15}$ or below at an averaging time less than one day [1], [2], [3]. For primary frequency standards realizing the SI second, it is necessary to compare their frequencies to ensure the accuracy. Two-Way Satellite Time and Frequency Transfer (TWSTFT) and GPS carrier phase time transfer (GPSCP) [4] are state-of-the-art techniques to compare remotely located frequency standards. In fact, comparisons between primary frequency standards of several institutes in Europe and USA were performed at the $10^{-15}$ uncertainty level using these methods [5], [6]. National Institute of Information and Communications Technology (NICT) and Physikalisch-Technische Bundesanstalt (PTB) have established a long baseline TWSTFT link between Asia and Europe to contribute to the calculation of International Atomic Time (TAI) and to enable comparison between primary frequency standards [7]. In this report, the performance of the TWSTFT link is described briefly. The results of the fountain comparison between NICT and PTB by GPSCP and GPS P3 All-in-View (P3AV) [8] as well as TWSTFT are presented.

\section{TWSTFT LINK BETWEEN NICT AND PTB}

In July 2005, the TWSTFT link between Asia and Europe was established. With the use of two transponders on the geostationary satellite PAS-4 a baseline of $8300 \mathrm{~km}$ is bridged. A multi-channel TWSTFT modem developed by NICT is used and provides a continuous data stream with one measurement made every second [9]. For analysis, the TWSTFT data of 5 minutes every hour are processed following the recommendation ITU-R TF. 1153-2 [10]. The data have been used in the TAI calculation since April 2007.

In Fig. 1, circles and squares show the frequency instability of UTC(NICT)-UTC(PTB) obtained by TWSTFT and P3AV, respectively. Data observation period is from $2006 / 2 / 7$ to

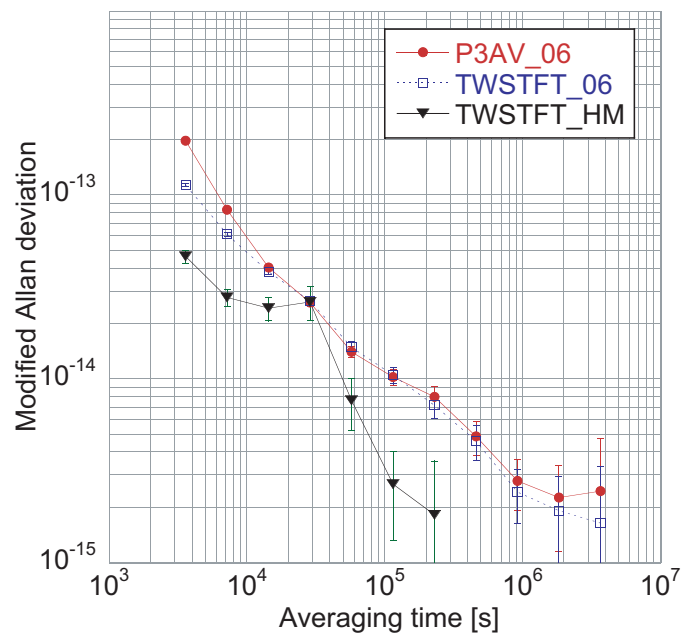

Fig. 1. Frequency instability of UTC(NICT)-UTC(PTB).

2006/11/30. While short term stabilities by TWSTFT are slightly better than those by $\mathrm{P} 3 \mathrm{AV}$, for averaging times longer than $10^{4} \mathrm{~s}$ the instability is that of UTC(PTB) because it is realized by the thermal beam primary caesium clock CS2 [11]. To examine the link performance, the reference signals from H-masers were connected with the TWSTFT systems in both stations. The measurement using the H-masers was performed for 16 days starting from 2006/12/9. The corresponding frequency instability is depicted in Fig. 1 as triangles. Compared with the previous results, it proved that short and long term stabilities in the case of the H-masers are better than those of UTC(NICT)-UTC(PTB). The instabilities around the averaging times of $3 \times 10^{4} \mathrm{~s}$ have a bump, however. It is due to a diurnal variation and we have already reported on studies regarding their reason [12]. So far ionospheric and internal delays in the earth stations seem to explain a small part of the observed diurnals only. Fig. 2 shows time differences between the H-masers in both institutes. Red line and blue dots represent 1-hour and 1-day averaged data, respectively. The diurnal variation is almost removed by 1-day averaging. The frequency stability at 1-day average is about $3 \times 10^{-15}$, which is sufficient for a comparison between fountain primary frequency standards. 


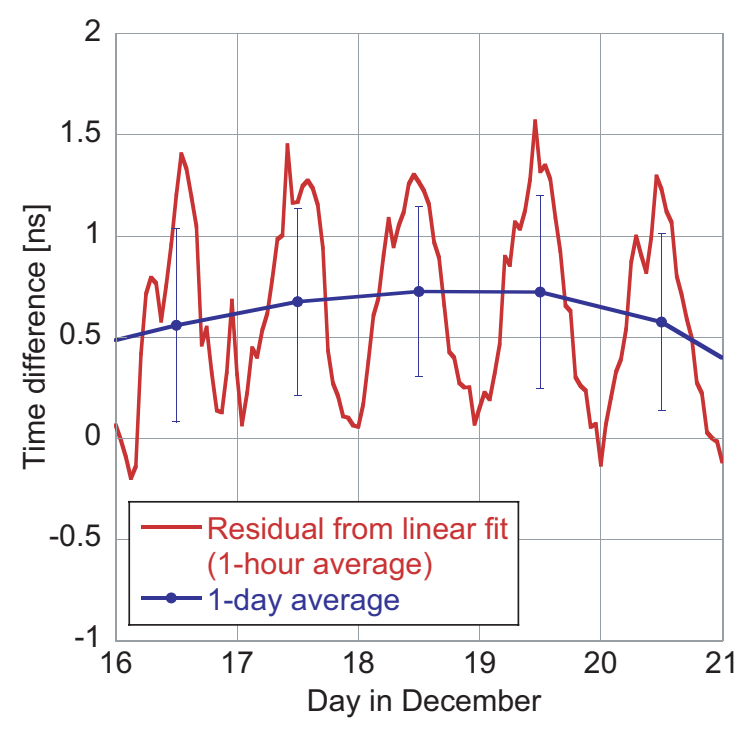

Fig. 2. Time difference between H-masers. Frequency offset is removed.

\section{COMPARISON OF PRIMARY FREQUENCY STANDARDS}

\section{A. Comparison setup}

In frequency comparisons between caesium atomic fountains, the frequency difference between the fountain and the $\mathrm{H}$-maser used as a reference for the fountain is measured in each laboratory. Then the frequency difference between the $\mathrm{H}$-masers is determined by remote frequency transfer. Combining them, the frequency difference between the fountains is derived. This is done by computing their mean differences calculated in a straightforward manner, $(\bar{T}($ end $)-\bar{T}($ start $))$ and divided by the measurement interval.

PTB and NICT simultaneously operated their caesium atomic fountain primary frequency standards, PTB-CSF1 and NICT-CsF1 for 15 days starting from 2007/12/10. In PTB, the reference $\mathrm{H}$-maser is named $\mathrm{H} 5$ and NICT names the reference $\mathrm{H}$-maser as HM8. The measurement configuration is depicted in Fig. 3. The frequency difference between H5 and HM8 was determined directly by TWSTFT. The following equation shows the frequency difference via TWSTFT between the fountains. Each term in parenthesis represents a frequency difference.

$$
\begin{array}{rll}
(\mathrm{PTB}-\mathrm{CSF} 1 & - & \text { NICT-CsF1 })_{\text {via }} \mathrm{TW} \\
=(\mathrm{PTB}-\mathrm{CSF} 1-\mathrm{H} 5) & - & (\mathrm{NICT}-\mathrm{CsF} 1-\mathrm{HM} 8) \\
& +(\mathrm{H} 5-\mathrm{HM} 8)_{\text {via }} \mathrm{TW} .
\end{array}
$$

To compare the result with other frequency transfer methods, the results were calculated using GPS data and Circular T [13]. GPS receivers, Ashtech Z-12T and Septentrio PolaRx2 in PTB and NICT, respectively, are used in the international time links organized by BIPM. The frequency difference between UTC(PTB) and UTC(NICT) was determined by P3AV and GPSCP, whose detailed analysis method is described in [14]. In December 2006, the TAI time link between PTB and
NICT was maintained by P3AV. At PTB the phase difference between UTC(PTB) and H5 was measured by a phase comparator. At NICT, a time interval counter was used for the phase difference measurement between UTC(NICT) and HM8. Then the frequency differences between the fountains via GPSCP and P3AV were calculated in the following way:

$$
\begin{aligned}
(\text { PTB-CSF1 } & - \text { NICT-CsF1 })_{\text {via GPS }} \\
=(\text { PTB-CSF1 }-\mathrm{H} 5) & -(\text { NICT-CsF1 }- \text { HM8 }) \\
& +(\text { H5 }- \text { HM8 })_{\text {via GPS }}
\end{aligned}
$$

and

$$
\begin{aligned}
& \text { (H5 - HM8) })_{\text {via GPS }} \\
& =(\mathrm{UTC}(\mathrm{NICT})-\mathrm{HM} 8)-(\mathrm{UTC}(\mathrm{PTB})-\mathrm{H} 5) \\
& +(\mathrm{UTC}(\mathrm{PTB})-\mathrm{UTC}(\mathrm{NICT}))_{\text {via GPS }} .
\end{aligned}
$$

In Circular T, time differences with respect to Coordinated Universal Time (UTC) with a five-days grid are reported. The operation result of PTB-CSF1 for this 15 days was reported to BIPM and the frequency difference between PTB-CSF1 and TAI was calculated there and published in the Circular T 228. We computed the frequency difference of TAI - UTC(NICT) using the values of the Circular T 228. Then the mean H-maser difference with respect to UTC(NICT) was calculated and the frequency difference between the fountains via the Circular $\mathrm{T}$ was obtained as follows:

$$
\begin{array}{rll}
(\text { PTB-CSF1 } & - & \text { NICT-CsF1 })_{\text {via }} \text { irT } \\
=(\text { PTB-CSF1 }- \text { TAI }) & - & (\text { NICT-CsF1 }- \text { HM8 }) \\
+(\mathrm{UTC}(\text { NICT }) & - & \text { HM8 }) \\
+(\text { TAI } & - & \text { UTC }(\text { NICT }))_{\text {via CirT }} .
\end{array}
$$

\section{B. Results}

The H5-HM8 data via TWSTFT were achieved every second. While the phase differences between UTC(PTB) and H5 were taken every ten minutes by a phase comparator, those between UTC(NICT) and HM8 were measured every second by a time interval counter. The time differences of UTC(PTB)UTC(NICT) via P3AV and GPSCP were calculated every 16 min and $10 \mathrm{~min}$, respectively. Then all the data were averaged per hour and adopted for the computation of the frequency transfer. Fig. 4 shows the daily frequency difference between H5 and HM8 via TWSTFT, GPSCP and P3AV. The frequency differences per day were computed from the average of 24 hourly frequency differences per day. Consequently, the daily difference is equal to that derived from the straightforward manner using the time comparison data of $0 \mathrm{~h}$ and $24 \mathrm{~h}$. From the plot one can infer that the results via three different methods show almost the same linear trend, which means that the frequency transfers connecting with the H-masers and local UTC work well. To estimate the frequency difference between the $\mathrm{H}$-masers, we assumed that it was a constant over this period and fluctuation in each day around the mean frequency over 15 days was induced by phase noise due to 


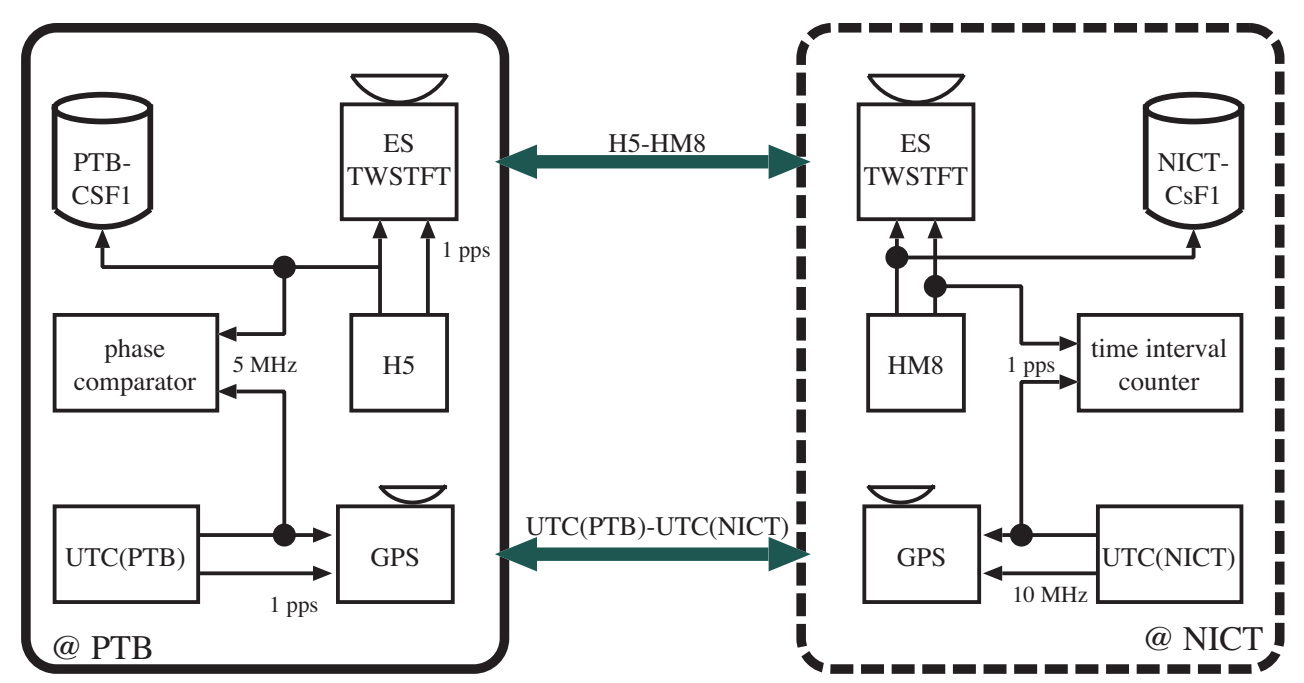

Fig. 3. Frequency comparison setup. ES represents earth station.

the link. The phase noise corresponds to the link uncertainty at 1 day. If the frequencies of the H-masers vary, the phase noise is overestimated, which is consistent with the link noise of $0.5 \mathrm{~ns}$ assigned by BIPM for TWSTFT, however. A detailed discussion is described in the next section. Since the measurement is performed for 15 days, it is necessary to extend the uncertainty for 15 days. We used the extension method approved in the 17th CCTF meeting [16]. When a frequency comparison in a link with a measurement interval of $\tau_{0}$ continues for $\tau$, the link uncertainty $u$ is written as:

$$
u=\left(\frac{\sqrt{2} u_{\mathrm{A}}}{\tau_{0}}\right) \frac{1}{n^{x}},
$$

$u_{r_{m} A}$ is the phase noise of the link and $n=\tau / \tau_{0} . x=0.9$ is used in the calculation of the frequency transfer instability into TAI for primary frequency standards. This is decided by the assumption that the link noise would be mainly due to white $\mathrm{PM}$ and flicker PM in the range from 1 day to many tens of days. We estimated the link instabilities for 15 days using this equation. In the case via Circular T, the link uncertainties were computed from the type-A uncertainty $u_{\mathrm{A}}$ of the realization of local UTC. For PTB and NICT, $u_{\mathrm{A}}$ were $0.3 \mathrm{~ns}$ and $0.7 \mathrm{~ns}$ for this period, respectively. Their root sum square was adopted for the link uncertainty. The H-maser differences and the link uncertainties are summarized in Table I.

The detailed uncertainty budget for the fountains were described in [1], [2], [3] and in the report submitted to BIPM [15]. There, $u_{\mathrm{A}}$ and $u_{\mathrm{B}}$ represent the combined uncertainties due to the statistical instability and systematic effects, respectively. $u_{\text {tot }}$ shows the total uncertainty of the fountains, excluding the link uncertainty. Uncertainties of PTB-CSF1 and NICT-CsF1 are summarized in Table II.

The mean frequency differences between PTB-CSF1 and NICT-CsF1 via TWSTFT, GPSCP, P3AV and the Circular $\mathrm{T}$ are summarized in Table III. PTB-CSF1 and NICT-CsF1 show good agreement within the stated uncertainties. The values of $u_{\mathrm{A}}$ and $u_{\text {tot }}$ represent the type $\mathrm{A}$ and the combined uncertainties including the link uncertainty.

TABLE I

FREQUENCY DIFFERENCES BETWEEN H5 AND HM8 DURING 15 DAYS.

\begin{tabular}{lccc}
\hline & H5 - HM8 $\left[\times 10^{-15}\right]$ & $u_{\mathrm{A}}[\mathrm{ps}]$ & $u\left[\times 10^{-15}\right]$ \\
\hline \hline TWSTFT & -95.0 & 508 & 0.7 \\
GPSCP & -94.9 & 428 & 0.6 \\
P3AV & -94.5 & 544 & 0.8 \\
Circular T & -94.1 & 762 & 1.0 \\
\hline
\end{tabular}

TABLE II

UNCERTAINTIES OF PTB-CSF1 AND NICT-CSF1 EXPRESSED IN $10^{-15}$.

\begin{tabular}{lccc}
\hline & $\mathrm{u}_{\mathrm{A}}$ & $\mathrm{u}_{\mathrm{B}}$ & $\mathrm{u}_{\text {tot }}$ \\
\hline \hline PTB-CSF1 & 1.0 & 1.1 & 1.5 \\
NICT-CsF1 & 1.4 & 1.9 & 2.4 \\
\hline
\end{tabular}

TABLE III

FREQUENCY DIFFERENCES BETWEEN PTB-CSF1 AND NICT-CSF1 DURING 15 DAYS STARTING FROM MJD 54079. NUMBERS ARE EXPRESSED IN $10^{-15}$

\begin{tabular}{lccc}
\hline & PTB-CSF1 - NICT-CsF1 & $u_{\mathrm{A}}$ & $u_{\text {tot }}$ \\
\hline \hline TWSTFT & -1.3 & 1.9 & 2.9 \\
GPSCP & -1.2 & 1.8 & 2.9 \\
P3AV & -0.8 & 1.9 & 2.9 \\
Circular T & -0.4 & 2.0 & 3.0 \\
\hline
\end{tabular}

\section{Discussion}

The assumption that the H-maser difference was a constant is discussed in this section. To evaluate the link uncertainty and noise, frequency transfer instability of the H-masers by three methods and double difference between them were calculated as shown in Fig. 5. The frequency instability obtained by TWSTFT has a bump around the averaging time of a half 


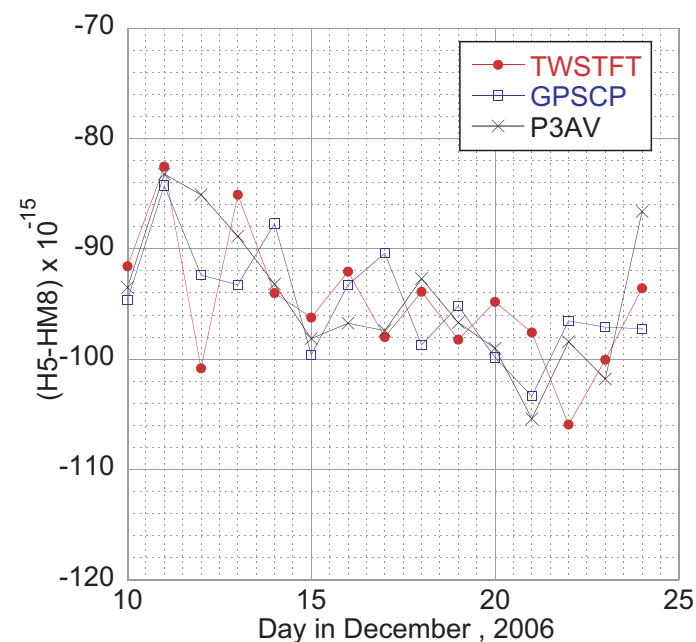

Fig. 4. Frequency difference between H-masers.

day. For shorter and longer averaging times, both instabilities by TWSTFT and GPSCP show good agreement. At short averaging times, the instability by GPSCP has a slope of about $\tau^{-3 / 2}$ representing white phase noise. A periodic component is not seen there. P3AV is inferior to the other methods and there is a small bump around a half day, too. Averaging longer than $2 \times 10^{5}$ seconds gives the same instability for all three methods, which might be due to frequency drifts of the H-masers. The frequency instability of the double difference, GPSCP-P3AV, is $0.7 \times 10^{-15}$ at 324000 seconds, equal to a quarter of 15 days. Because both the GPSCP and P3AV data were obtained from the same receivers, all common mode variations in the GPS receivers and frequency references are canceled in the differences. So, this instability attributes to the difference in the two types of the data processing software. On the other hand, the frequency instability of TWSTFT-GPSCP is nearly at the same level, $0.8 \times 10^{-15}$ at the time, being an upper limit on the TWSTFT instability. These results prove that the assumption about the H-maser difference and the estimated link uncertainties in Table I were almost appropriate. The modified Allan deviation of the TWSTFT-GPSCP looks like Flicker FM in the range of averaging times longer than $2 \times 10^{5}$ seconds, however. The link noise in the range over a few days should be examined more intensively.

When we computed the result of the fountain comparison via P3AV, the frequency difference of UTC(NICT)-UTC(PTB) was calculated first and then connected with the other results following the equations (2) and (3). The time link between PTB and NICT for TAI was connected by P3AV at that time. The frequency difference of UTC(NICT)-UTC(PTB) by the Circular $\mathrm{T}$ differed a little from the P3AV result of this work. This is why there is difference between the fountain comparison results via the Circular $\mathrm{T}$ and P3AV. A possible reason is a difference in the data processing. In the TAI computation, a low-pass filtering process is adopted to reduce white PM noise whose time constant is close to about 14 hours
[17]. On the other hand, we used a simple average in the data analysis, because the H-maser difference was smooth enough. Fig. 6 shows time and frequency differences of UTC(NICT)UTC(PTB) obtained by P3AV and the Circular T. There, thin red and black dotted lines represent 1-hour data and 14hours moving average data, respectively. Squares represent the values from the Circular T. Slopes of solid red, black and blue lines express the average frequency differences obtained from $12 / 10$ to $12 / 25$ by 1 -hour data, moving averaged P3AV data and the Circular T, respectively. Their frequency differences are summarized in Table IV. The result using the moving average was consistent with the Circular $\mathrm{T}$, but not with the 1-hour average, to be explained from the perspective of the data processing method. Further study about the disagreement with Circular $\mathrm{T}$ is required.

\section{TABLE IV}

FREQUENCY DIFFERENCE OF UTC(NICT)-UTC(PTB) DURING 15 DAYS. NUMBERS ARE EXPRESSED IN $10^{-15}$.

\begin{tabular}{lc}
\hline & Frequency difference \\
\hline \hline Circular T & -3.9 \\
P3AV (1-hour average) & -3.5 \\
P3AV (14-hours moving average) & -3.9 \\
\hline
\end{tabular}

\section{CONCLUSIONS}

PTB and NICT simultaneously operated their caesium atomic fountains, PTB-CSF1 and NICT-CsF1 for 15 days starting from 2007/12/10. PTB-CSF1 and NICT-CsF1 agree within the stated uncertainty and the results via TWSTFT, GPSCP, P3AV and Circular T were consistent. It was the first frequency comparison between Europe and Asia of primary caesium fountains. The frequency difference between the local reference H-masers was determined directly in the TWSTFT link with an uncertainty level below $10^{-15}$. As another result, the combined uncertainty was smaller than that via the Circular T. Direct links are preferable because they are easier to characterize in terms of the the actual link conditions and that gives the possibility to make the link uncertainty smaller.

\section{ACKNOWLEDGMENT}

The authors would like to thank J. Becker for his support to the operation of the PTB station.

\section{REFERENCES}

[1] S. Weyers, U. Hübner, R. Schröder, C. Tamm, A. Bauch, "Uncertainty evaluation of the atomic caesium fountain CSF1 of the PTB", Metrologia 38, pp. 343-352, 2001.

[2] S. Weyers, A. Bauch, R. Schröder, C. Tamm, "The atomic caesium fountain CSF1 of PTB", Proc. 6th Symp. on Freq. Standards and Metrology, pp. 64-71, World Scientific, 2002.

[3] M. Kumagai, H. Ito, M. Kajita, M. Hosokawa, "NICT's operational atomic fountain NICT-CsF1", Proc. of Asia-Pacific Time and Frequency Forum, pp. 77-83, 2006.

[4] R. Dach et al., "Time transfer using GPS carrier phase: error propagation and results", Journal of Geodesy, DOI 10.1007/s00190-002-0296-z, 2003.

[5] T. Parker et al., "First comparison of remote cesium fountains", Proc. of Frequency Control Symposium, pp. 63-68, 2001.

[6] A. Bauch et al., "Comparison between frequency standards in Europe and the USA at the $10^{-15}$ uncertainty level", Metrologia 43, pp. 109-120, 2006. 

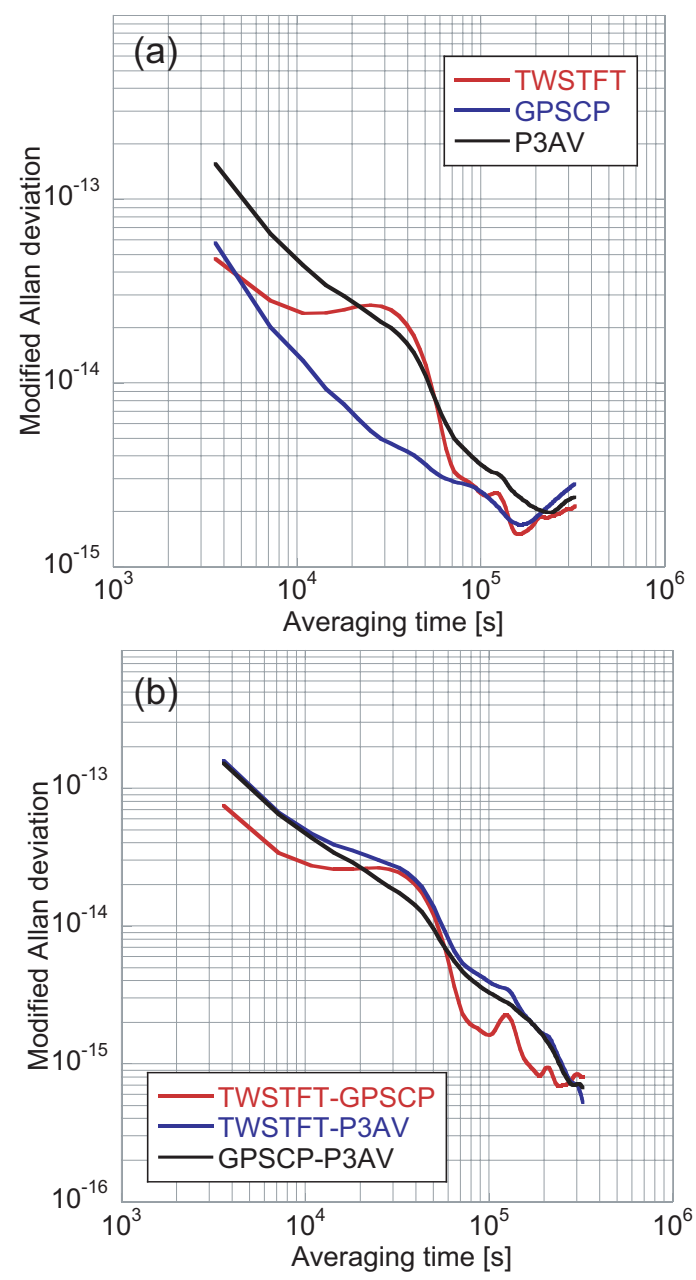

Fig. 5. Frequency instabilities of (a) H5-HM8 and (b) double differences among TWSTFT, GPSCP and P3AV.

[7] H. Maeno et al., "Establishment of a TWSTFT link between Asia and Europe connecting NICT and PTB", Proc. of 20 th European Frequency and Time Forum, pp. 575-579, 2006.

[8] Z. Jiang and G. Petit, "Time transfer with GPS satellite all in view", Proc. of ATF 2004, pp. 236-243, Beijing, China, 2004.

[9] M. Fujieda, M. Aida, H. Maeno, L. Tung, J. Amagai, "Delay difference calibration of TWSTFT earth station using multi-channel modem", IEEE Trans. Inst. Meas., vol. 56, No. 2, pp. 346-350, 2007.

[10] Recommendation ITU-R TF. 1153-2, Geneva, last update 2003.

[11] A. Bauch, "The PTB primary clocks CS1 and CS2", Metrologia 42, S43-S54, 2005.

[12] M. Fujieda et al., "Long baseline TWSTFT between Asia and Europe", Proc of 38th PTTI, 2006, in press.

[13] Circular T no.228, ftp://ftp2.bipm.org/pub/tai/publication/cirt.228

[14] T. Gotoh, M. Fujieda, J. Amagai, "Comparison study of GPS carrier phase and Two-way satellite time and frequency transfer", in these proceedings.

[15] ptb-csf1_54079-54094.pdf, ftp://ftp2.bipm.org/pub/tai/data/PFS_reports/

[16] T. E. Parker, "Report to the 17th Session of the CCTF", Conference report, CCTF/06-13, 2006.

[17] G. Petit and Z. Jiang, "Long-term comparison of GPS P3 links with other techniques used for TAI", BIPM report, GP/TIM.128, December, 2003.

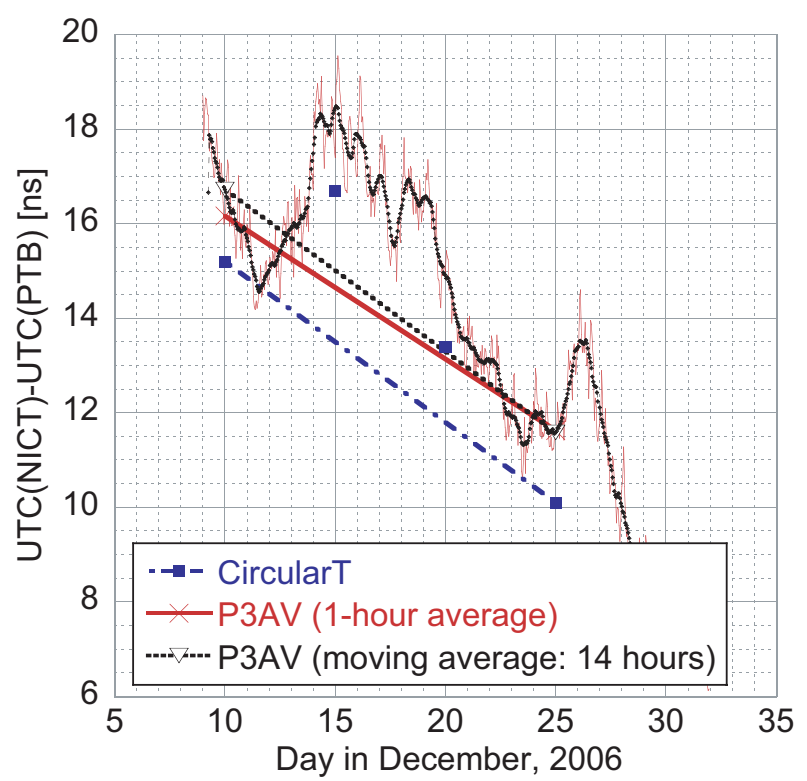

Fig. 6. Time difference of UTC(NICT)-UTC(PTB) by P3AV. Thin red and black dotted lines represent 1-hour data and 14-hours moving averaged data, respectively. Squares show the values from the circular T. Gradients of solid red, black and blue lines represent the frequency differences during 15 days. 